\title{
The Simulation and Minimization of Loss Probability in the Tandem Queueing with Two Heterogeneous Channels
}

\author{
Müjgan Zobu, Vedat Sağlam, Murat Sağır, Erdinç Yücesoy, and Tolga Zaman \\ Department of Statistics, Faculty of Science and Arts, Ondokuz Mayıs University, Kurupelit, 55100 Samsun, Turkey \\ Correspondence should be addressed to Müjgan Zobu; mujganzobu@hotmail.com
}

Received 11 July 2013; Accepted 3 October 2013

Academic Editor: Hao Shen

Copyright ( 2013 Müjgan Zobu et al. This is an open access article distributed under the Creative Commons Attribution License, which permits unrestricted use, distribution, and reproduction in any medium, provided the original work is properly cited.

\begin{abstract}
A stochastic model consisting of two heterogeneous channels and having no waiting room in front of each is considered. A customer who has completed his service in channel 1 while channel 2 is busy blocks channel 1 with probability $Q$ or leaves the system with $1-Q$ probability. This model was analysed: expected number of customer and loss probability of customer are calculated and optimal ordering of channels minimizing parameters has been found. Additionally, this model was simulated; furthermore, simulated and exact results of loss probabilities of customers were given in the tables.
\end{abstract}

\section{Introduction}

Due to the recent developments in the fields of production line and communication, new queuing theory models are needed. In this sense, significant improvements in serial channel models have been observed. A tandem queueing system consist of two stage with waiting in each stage considered and joint limit distribution of waiting times of $n$th customer in this system has been investigated in [1]. When all service times were fixed, it was showed that departure times do not affect the order of service channels, in [2]. It was demonstrated diagrammatically that optimal service parameters were $\mu_{1}=\mu_{2}=c / 2$ under the condition that $\mu_{1}+\mu_{2}=c$ in tandem system with two channels having unlimited number of customers in front of each channel and conforming interarrivals and service duration exponential distribution, in [3]. In [4], it was showed that optimal order of service in tandem system with two channels can be achieved when service time of one channel is fixed and placed in channel-1. It was demonstrated that departure times were stochastically faster when interarrival times are general, the lengths of service are exponential, and service times of channels for tandem queue with more than two single stations are equal, in [5]. The performance of twostage tandem queuing model with blocking was introduced in [6]. Loss probability of customers, the average number of customers in a system with heterogeneous channels having no waiting rooms were obtained; also optimal values of loss probability of customers, the average number of customers were calculated in [7]. The discrete-time tandem queuing model based on Markov processes was analysed in [8]. A new queuing model with sequential two stations (stages), single server at each station, where no queue is allowed at station 2 and with no restriction at station 1, was introduced in [9]. There is a FCFS service discipline in which the input stream is Poisson having rate $\lambda$. The service time of any customer at server $i(i=1,2)$ is exponential with parameter $\mu_{i}$. The state probabilities and loss probability of this model are given. The performance measures are obtained and optimized, and, additionally, the model is simulated.

In this study, we discuss a tandem model consisting of two stages channels: a stochastic queueing model consisting of two heterogeneous channels and having no waiting room in front of each is considered. A customer who has completed his service in channel 1 while channel 2 is busy blocks channel-1 with probability $Q$ or leaves the system with probability $1-Q$. The measures of performance of this model for $Q$ and loss probabilities of customers are calculated and optimal ordering of channels has been found. Additionally, this model was simulated and compared with the actual results. A two-stage blocking tandem queueing system with no waiting in front of each stage is analysed in [10]. We 


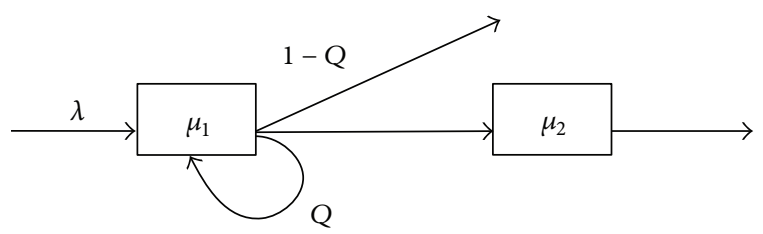

FIGURE 1: The tandem model with blocking.

have obtained the general form of the model given in [10] depending on $Q$. If $Q=1$ is taken in our model, the model in [10] is obtained. While there is generally waiting line in the service systems, because of obligations and urgency and unavailability of desired features, waiting line may not occur as in our proposed model. Therefore, we have decided to construct and analyze such a model it.

\section{Definition of the Model}

This model is defined as follows: a customer completes his service in channel 1 (when this channel is empty), enters into channel 2 (when channel two is empty), and leaves the system after receiving service. The customer, who has completed his service in channel 1, while channel 2 is busy, blocks channel 1 with probability $Q$ or leaves the system with probability $1-Q$. Interarrival times and service times in channel 1 and channel 2 are assumed to be independent and have exponential distribution with $\lambda, \mu_{1}$, and $\mu_{2}$ parameters respectively. This model is shown in Figure 1.

2.1. State Probabilities. Channel 1 can be empty, busy, or blocked. Let them be represented them with 0,1 , and $b$, respectively. Channel 2 can be 0 or 1 . Let $\xi_{t}$ be the state of channel 1 and $\eta_{t}$ the state of channel 2 at any $t$ time. In this case, $\left\{\left(\xi_{t}, \eta_{t}\right), t \geq 0\right\}$ is a two-dimensional Markov chain with continuous parameter and state space

$$
\begin{gathered}
E=\{(0,0),(0,1),(1,0),(1,1),(b, 1)\}, \\
P_{i j}(t)=\operatorname{Prob}\left\{\xi_{t}=i, \eta_{t}=j\right\}, \quad \forall(i, j) \in E .
\end{gathered}
$$

Kolmogorov differential equation for these probabilities is obtained. The transient probabilities of the process $\left\{\left(\xi_{t}, \eta_{t}\right), t \geq 0\right\}$ will be found for $(t, t+h)$ as $h \rightarrow 0$, namely,

$$
P_{i j}^{\prime}(t)=\sum_{k \in E} P_{i k}(t) a_{k j}
$$

Furthermore, it is supposed that limit probabilities of $P_{i j}(t)$ are existing as follows:

$$
\begin{gathered}
\lim _{t \rightarrow \infty} P_{i j}(t)=p_{i j}, \\
\lim _{t \rightarrow \infty} P_{i j}^{\prime}(t)=\sum_{k \in E} \lim _{t \rightarrow \infty} P_{i k}(t) a_{k j}=\sum_{k \in E} p_{i k} a_{k j}=0
\end{gathered}
$$

where $a_{i j}$ is transition rates and matrix of transition rates $\left(a_{i j}\right)$ is shown as follows:

$$
\begin{gathered}
\mathrm{A}=\left(\begin{array}{ccccc}
-\lambda & 0 & \lambda & 0 & 0 \\
\mu_{2} & -\left(\lambda+\mu_{2}\right) & 0 & \lambda & 0 \\
0 & \mu_{1} & -\mu_{1} & 0 & 0 \\
0 & \mu_{1}(1-Q) & \mu_{2} & -\left(\mu_{1}+\mu_{2}\right) & \mu_{1} Q \\
0 & \mu_{2} & 0 & 0 & -\mu_{2}
\end{array}\right), \\
\sum_{j} a_{i j}=0, \quad a_{i i} \leq 0, \quad a_{i j} \geq 0, \quad \forall i, j \in E \\
\sum_{(i, j) \in E} p_{i j}=1 .
\end{gathered}
$$

Steady-state equations for $\left\{\left(\xi_{t}, \eta_{t}\right), t \geq 0\right\}$ from equality (4) are obtained follow as

$$
\begin{gathered}
0=-\lambda p_{00}+\mu_{2} p_{01}, \\
0=-\left(\lambda+\mu_{2}\right) p_{01}+\mu_{1} p_{10}+\mu_{1}(1-Q) p_{11}+\mu_{2} p_{b 1}, \\
0=\lambda p_{00}-\mu_{1} p_{10}+\mu_{2} p_{11}, \\
0=\lambda p_{01}-\left(\mu_{1}+\mu_{2}\right) p_{11}, \\
0=\mu_{1} Q p_{11}+\mu_{2} p_{b 1} .
\end{gathered}
$$

We define $r_{i}=\lambda / \mu_{i}$ for $i=1,2$ and $r=\lambda /\left(\mu_{1}+\mu_{2}\right)$.

If we solve this steady-state equations, we obtain the $p_{i j}$ probabilities as follows:

$$
\begin{array}{ll}
p_{01}=r_{2} p_{00}, & p_{10}=r_{1}(1+r) p_{00}, \\
p_{11}=r r_{2} p_{00}, & p_{b 1}=\frac{r_{2}^{2} r Q p_{00}}{r_{1}} .
\end{array}
$$

From equality (7),

$$
p_{00}=\frac{1}{1+r_{2}+r_{1}(1+r)+r_{2} r+r_{2}^{2} r Q / r_{1}}
$$

is obtained.

\section{The Measures of Effectiveness}

3.1. Loss Probability. Since there is no queue in the system,

$$
\begin{aligned}
p_{\text {loss }} & =p_{10}+p_{11}+p_{b 1}=1-p_{00}-p_{01} \\
& =\frac{r_{1}(1+r)+r_{2} r+r_{2}^{2} r Q / r_{1}}{1+r_{2}+r_{1}(1+r)+r_{2} r+r_{2}^{2} r Q / r_{1}} .
\end{aligned}
$$

Theorem 1. The $p_{\text {loss }}$ probability is minimum for $Q=0$.

Proof. Equality (11) defines $p_{\text {loss }}$ probability as a function of $Q$. We will write equality (11) as follows:

$$
\frac{1}{p_{\text {loss }}}=1+\frac{1+r_{2}}{r_{1}(1+r)+r_{2} r+r_{2}^{2} r Q / r_{1}} .
$$

The maximum value of $1 / p_{\text {loss }}$ is minimum value of $p_{\text {loss }}$ where $0<p_{\text {loss }} \leq 1$. In equality (12), if we substitute 
TABLE 1: Loss probabilities for $\mu_{1}=2, \mu_{2}=2$.

\begin{tabular}{lcccccccc}
\hline \multirow{2}{*}{ Number of iteration } & \multicolumn{2}{c}{$Q=0$} & \multicolumn{2}{c}{$Q=0.4$} & \multicolumn{2}{c}{$Q=0.8$} & \multicolumn{2}{c}{$Q=1$} \\
& $\operatorname{Pr}_{\text {sim }}$ & $\operatorname{Pr}_{\text {exact }}$ & $\operatorname{Pr}_{\text {sim }}$ & $\operatorname{Pr}_{\text {exact }}$ & $\operatorname{Pr}_{\text {sim }}$ & $\operatorname{Pr}_{\text {exact }}$ & $\operatorname{Pr}_{\text {sim }}$ & $\operatorname{Pr}_{\text {exact }}$ \\
\hline 4000 & 0.749739 & 0.666667 & 0.621271 & 0.77551 & 0.605388 & 0.867925 & 0.597085 & 0.90991 \\
6000 & 0.749664 & 0.666667 & 0.636181 & 0.77551 & 0.621225 & 0.867925 & 0.613301 & 0.90991 \\
20000 & 0.749629 & 0.666667 & 0.740998 & 0.77551 & 0.731758 & 0.867925 & 0.726884 & 0.90991 \\
\hline
\end{tabular}

TABLE 2: Loss probabilities for $\mu_{1}=2, \mu_{2}=4$.

\begin{tabular}{|c|c|c|c|c|c|c|c|c|}
\hline \multirow{2}{*}{ Number of iteration } & \multicolumn{2}{|c|}{$Q=0$} & \multicolumn{2}{|c|}{$Q=0.4$} & \multicolumn{2}{|c|}{$Q=0.8$} & \multicolumn{2}{|c|}{$Q=1$} \\
\hline & $\operatorname{Pr}_{\text {sim }}$ & $\operatorname{Pr}_{\text {exact }}$ & $\operatorname{Pr}_{\text {sim }}$ & $\operatorname{Pr}_{\text {exact }}$ & $\operatorname{Pr}_{\text {sim }}$ & $\operatorname{Pr}_{\text {exact }}$ & $\operatorname{Pr}_{\text {sim }}$ & $\mathrm{Pr}_{\text {exact }}$ \\
\hline 4000 & 0.742478 & 0.666667 & 0.733363 & 0.71739 & 0.723433 & 0.765957 & 0.718256 & 0.78947 \\
\hline 6000 & 0.738897 & 0.666667 & 0.729511 & 0.71739 & 0.719331 & 0.765957 & 0.713901 & 0.78947 \\
\hline 20000 & 0.713914 & 0.666667 & 0.702537 & 0.71739 & 0.690275 & 0.765957 & 0.683827 & 0.78947 \\
\hline
\end{tabular}

$Q=0,1 / p_{\text {loss }}$ is maximum; that is $p_{\text {loss }}$ is minimum. These equalities are denoted in (13) and (14), respectively:

$$
\begin{gathered}
\frac{1}{p_{\text {loss }}^{(1)}}=1+\frac{1+r_{2}}{r_{1}(1+r)+r_{2} r}, \\
p_{\text {loss }}^{(1)}=\frac{r_{1}(1+r)+r_{2} r}{1+r_{2}+r_{1}(1+r)+r_{2} r}, \\
p_{\text {loss }}^{(1)} \leq p_{\text {loss }} .
\end{gathered}
$$

Consequently, the loss probability is minimum when blocking is cancelled.

3.2. The Mean Number of Customers. Let $N$ be the random variable that describes the number of customers in the system

$$
E(N)=\sum_{i} \sum_{j}(i+j) p_{i j} .
$$

From equality (9) and equality (10),

$$
E(N)=\frac{r_{2}+r_{2} r+2 r_{1}(1+r)+2 Q r_{2}^{2} r / r_{1}}{1+r_{2}+r_{1}(1+r)+r_{2} r+Q r_{2}^{2} r / r_{1}}
$$

Provided that we get $Q=0$ in equality (17) as result of Theorem 1, we can rewrite this equality as follows:

$$
E\left(N^{\prime}\right)=\frac{r_{2}+r_{2} r+2 r_{1}(1+r)}{1+r_{2}+r_{1}(1+r)+r_{2} r} .
$$

\section{Optimal Order of Channels}

Considering $Q=0$ optimization, let $C_{1}$ denote channel 1 and $C_{2}$ denote the channel 2 . When we place $C_{1}$ channel to the first station and $C_{2}$ channel to the second station, loss probability of a customer is $p_{\text {loss }}^{(1)}$. Similarly, when we place $C_{2}$ channel to the first station and $C_{1}$ channel to the second station, loss probability of a customer is $p_{\text {loss }}^{(2)}$, assuming

$$
\frac{1}{p_{\text {loss }}^{(2)}}=1+\frac{1+r_{1}}{r_{2}(1+r)+r_{1} r} \text {. }
$$

Suppose that $C_{1}$ channel to the first station and, respectively, $C_{2}$ channel to the second station is optimal ordering, in other words, it is $p_{\text {loss }}^{(1)} \leq p_{\text {loss }}^{(2)}$ for this order. When the channels are changed, it is

$$
\frac{1}{p_{\text {loss }}^{(1)}} \geq \frac{1}{p_{\text {loss }}^{(2)}}
$$

Substituting the equality (13) and equality (19) in inequality (20), $\mu_{1} \geq \mu_{2}$ is obtained. Namely, this system runs faster when channel with larger parameter should be placed in station 1 .

\section{Numerical Technique and Simulation}

The simulation of queuing system requires keeping of event list to determine what will happen in the next step. Events in simulations occur at random times to imitate randomness in real life (we do not know precisely when a customer arrives or how long the service lasts). Obtaining randomness that is required to simulate real life is possible using "random numbers." In the system, simulation results obtained from Matlab program for different probabilities of blocking channel 1 are given in tables below. Depending on various probabilities of blocking $(Q=0, Q=0.4, Q=0.8, Q=1)$ and for 3 different iterations steps that are 40000, 6000 and 20000 loss probabilities were calculated. Moreover, all results of exact probabilities $\left(\operatorname{Pr}_{\text {exact }}\right)$ and simulation probabilities $\left(\operatorname{Pr}_{\text {sim }}\right)$ were given in Tables 1,2 , and 3 for $\mu_{i}(i=1,2)$. It has been seen that the simulation probabilities approximated to the exact probabilities.

\section{Conclusion}

In the study, the loss probability of the customer in two heterogeneous channels tandem system was calculated. Additionally, optimal orders of channels were obtained. Theoretically, simulation study supported obtained system results. Exact and simulation results of the loss probability were given in Tables 1, 2, and 3 for various $Q$ probabilities and service parameters $\mu_{1}$ and $\mu_{2}$. When the number of iterations of the 
TABLE 3: Loss probabilities for $\mu_{1}=4, \mu_{2}=2$.

\begin{tabular}{|c|c|c|c|c|c|c|c|c|}
\hline \multirow{2}{*}{ Number of iteration } & \multicolumn{2}{|c|}{$Q=0$} & \multicolumn{2}{|c|}{$Q=0.4$} & \multicolumn{2}{|c|}{$Q=0.8$} & \multicolumn{2}{|c|}{$Q=1$} \\
\hline & $\mathrm{Pr}_{\text {sim }}$ & $\operatorname{Pr}_{\text {exact }}$ & $\operatorname{Pr}_{\text {sim }}$ & $\operatorname{Pr}_{\text {exact }}$ & $\operatorname{Pr}_{\text {sim }}$ & $\operatorname{Pr}_{\text {exact }}$ & $\operatorname{Pr}_{\text {sim }}$ & $\operatorname{Pr}_{\text {exact }}$ \\
\hline 4000 & 0.701866 & 0.500000 & 0.692689 & 0.65094 & 0.682842 & 0.762295 & 0.677761 & 0.80769 \\
\hline 6000 & 0.695796 & 0.500000 & 0.687814 & 0.65094 & 0.679183 & 0.762295 & 0.674738 & 0.80769 \\
\hline 20000 & 0.653493 & 0.500000 & 0.653303 & 0.65094 & 0.653278 & 0.762295 & 0.653290 & 0.80769 \\
\hline
\end{tabular}

simulation is increased, theoretical and simulation results are observed being closer to each other. Furthermore, optimal ordering was confirmed for $Q=0, \mu_{1}=4$, and $\mu_{2}=2$ in Table 3. In other words, it is seen numerically in Table 3 that the loss probability of a customer is minimized when faster working channel is put in station 1 or optimal ordering is provided. In addition, it can be seen in every three tables that the loss probability of the customer is to increase as $Q$ values increase. If we get $Q=1$ in this model, model in [10] is obtained.

Finally, much more study can be done on this topic of interest by increasing the number of channels in the system.

\section{References}

[1] J. Sacks, "Ergodicity of queues in series," Annals of Mathematical Statistics, vol. 31, no. 3, pp. 579-588, 1960.

[2] H. D. Friedman, "Reduction methods for tandem queueing systems," Operations Research, vol. 13, pp. 121-131, 1965.

[3] R. B. Cooper, Introduction to Queueing Theory, Macmillan, New York, NY, USA, 1972.

[4] S. V. Tembe and R. W. Wolff, "The optimal order of service in tandem queues," Operations Research, vol. 22, no. 4, pp. 824-832, 1974.

[5] T. Lehtonen, "On the ordering of tandem queues with exponential servers," Journal of Applied Probability, vol. 23, no. 1, pp. 115129, 1986.

[6] A. Gómez-Corral and M. E. Martos, "Performance of two-stage tandem queues with blocking: the impact of several flows of signals," Performance Evaluation, vol. 63, no. 9-10, pp. 910-938, 2006.

[7] V. Savglam and H. Torun, "On optimization of stochastic service system with two heterogeneous channels," International Journal of Applied Mathematics, vol. 17, no. 1, pp. 1-6, 2005.

[8] X. Song and M. A. Mustafa, "A performance analysis of discretetime tandem queues with Markovian sources," Performance Evaluation, vol. 66, no. 9-10, pp. 524-543, 2009.

[9] V. Sağlam and M. Zobu, "A two-stage model queueing with no waiting line between channels," Mathematical Problems in Engineering, vol. 2013, Article ID 679369, 5 pages, 2013.

[10] F. Alpaslan, "On the minimization probability of loss in queue two heterogeneous channels," Pure and Applied Mathematika Sciences, vol. 43, no. 1-2, pp. 21-25, 1996. 


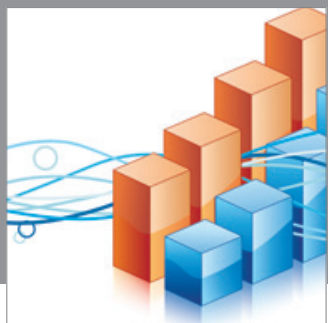

Advances in

Operations Research

mansans

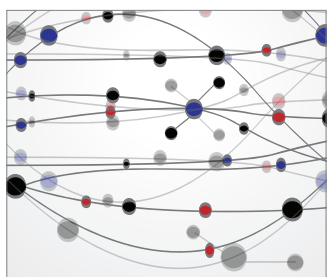

The Scientific World Journal
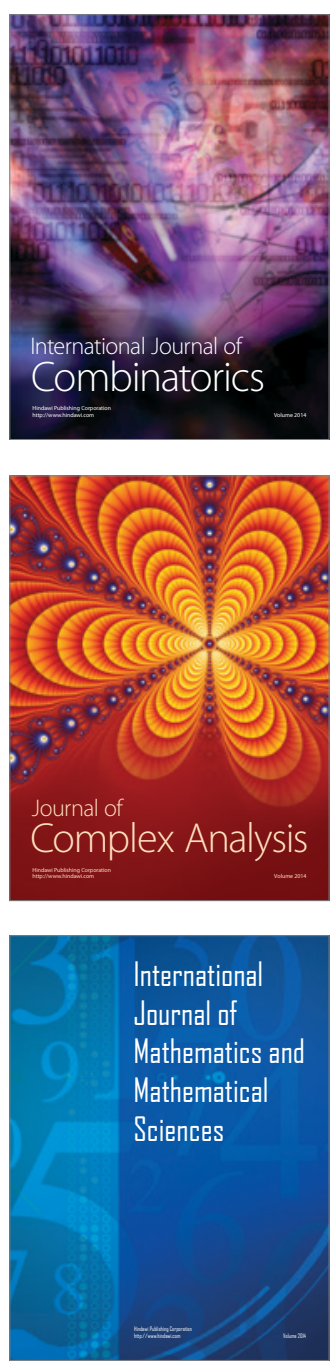
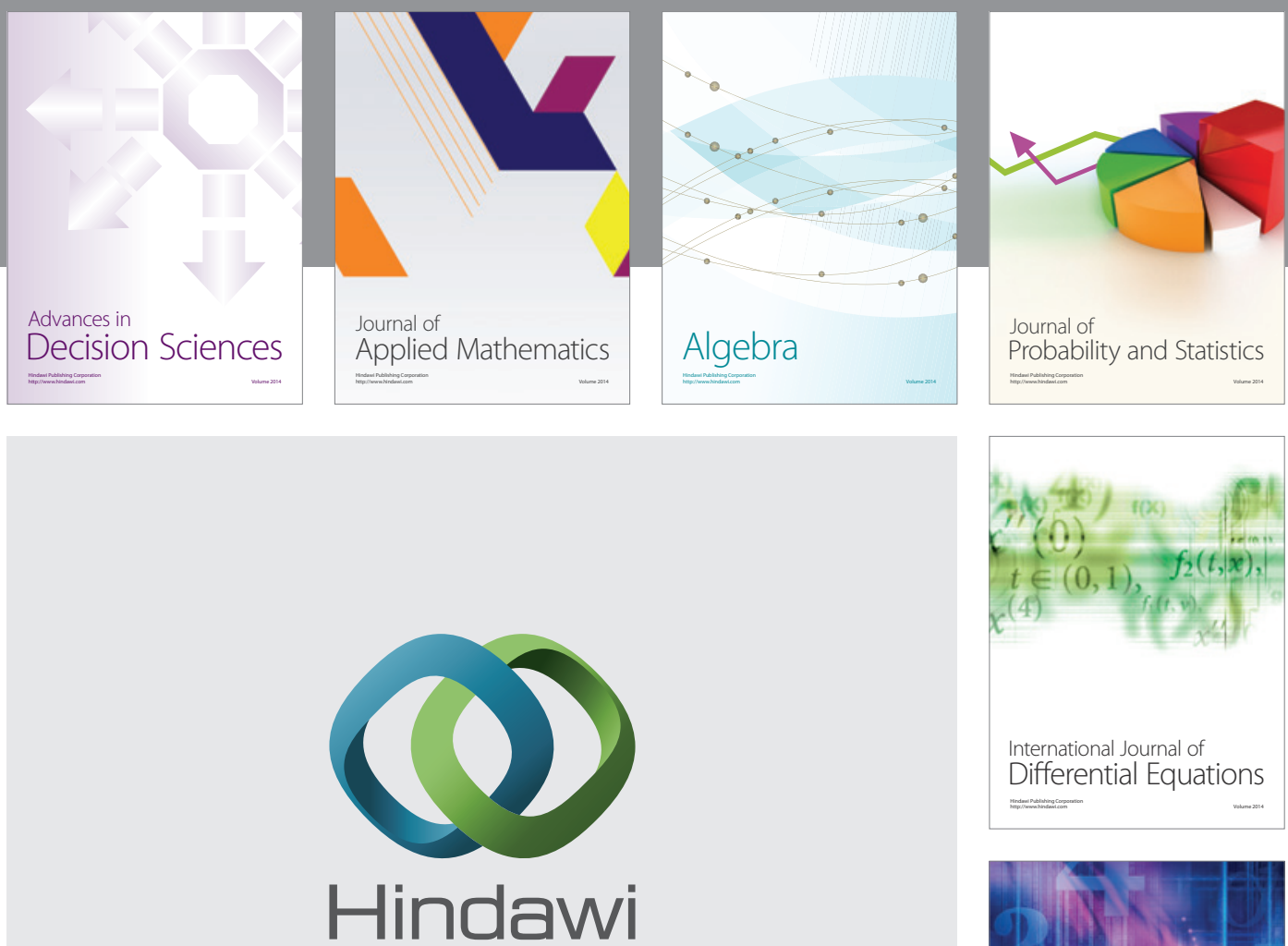

Submit your manuscripts at http://www.hindawi.com
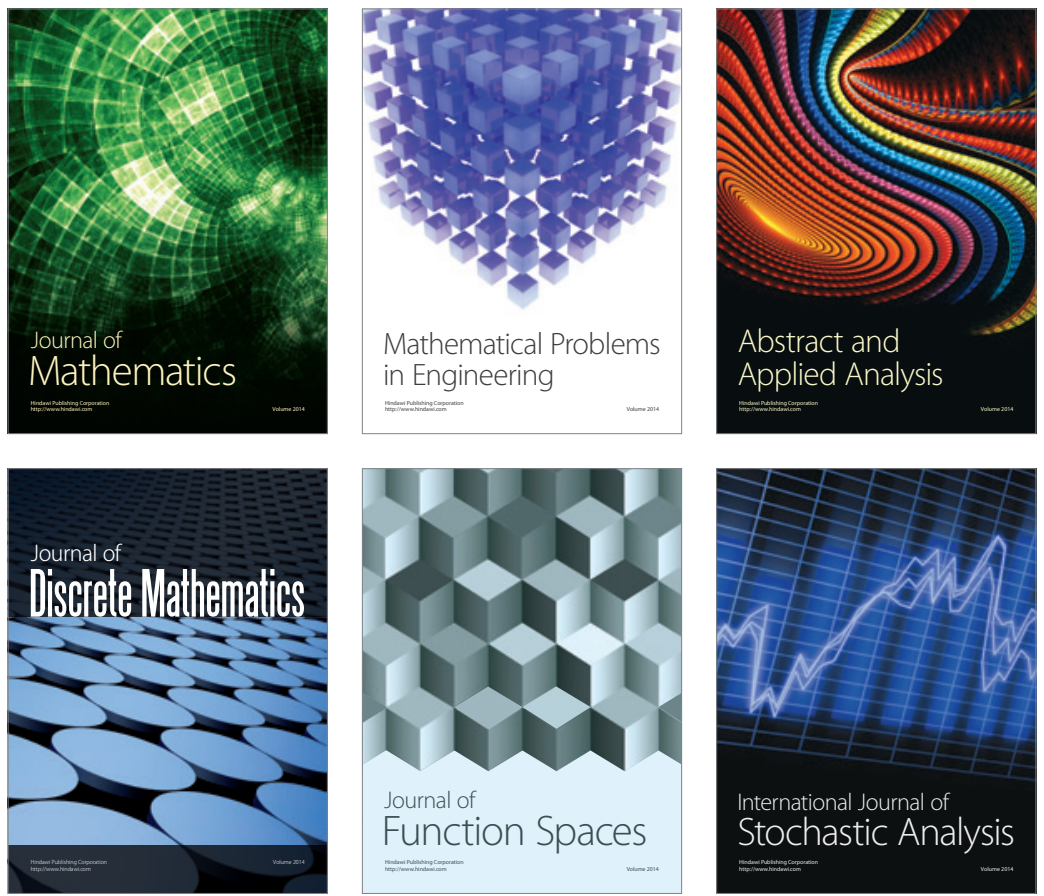

Journal of

Function Spaces

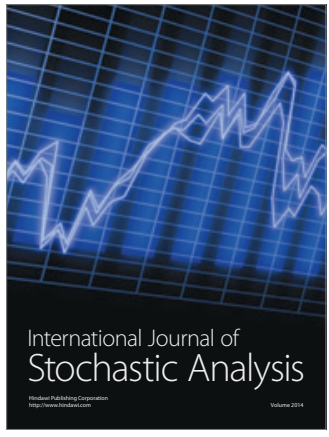

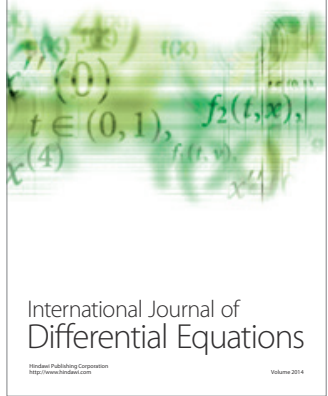
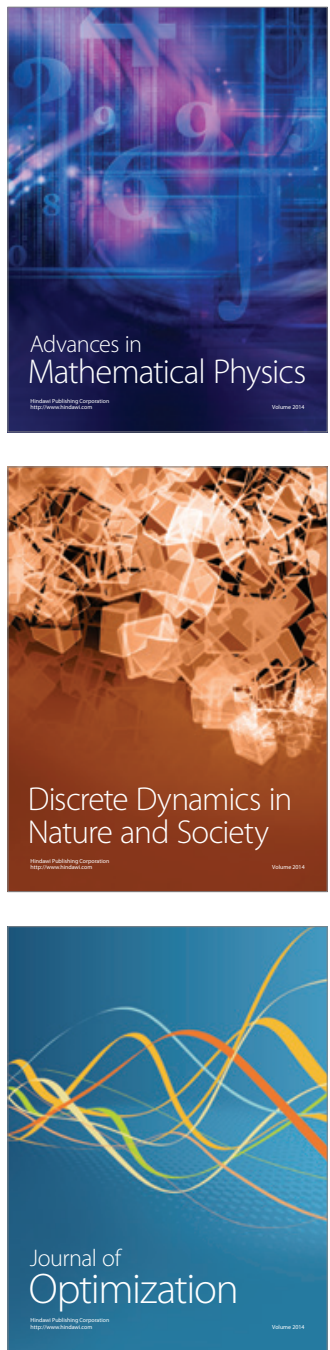